\title{
Measles virus in one-way crossing
}

It has long been assumed that the highly contagious measles virus (MV) enters its host through the luminal surface of respiratory epithelial cells before spreading systemically through the lymphatic system. A new paper published in The Journal of Clinical Investigation now reveals that initial infection of the airways is not necessary for entry into the host, but that airway infection is required for MV shedding and transmission.

The MV attachment protein haemagglutinin binds to signalling lymphocytic activation molecule (SLAM) on lymphatic cells. However, previous studies had suggested that SLAMdependent luminal entry does not occur immediately after infection, but rather that MV entry into the respiratory epithelium occurs basolaterally as the virus exits the host, and therefore that the virus uses an epithelial receptor (EpR) that is located on the basolateral surface of respiratory epithelial cells. To investigate the interactions between MV and this as-yet-unidentified EpR, Leonard, Cattaneo and colleagues used a structural model of MV haemagglutinin and mutational analyses to identify amino acid residues in haemagglutinin that are crucial for MV infection of epithelial cells, but are not required for infection of SLAM-expressing cells. Three residues were identified and the authors went on to generate mutant strains that remained dependent on SLAM for cell fusion, but were 'blind' to the EpR and were therefore unable to enter the airway epithelia. Rhesus monkeys that were inoculated with these strains intranasally developed

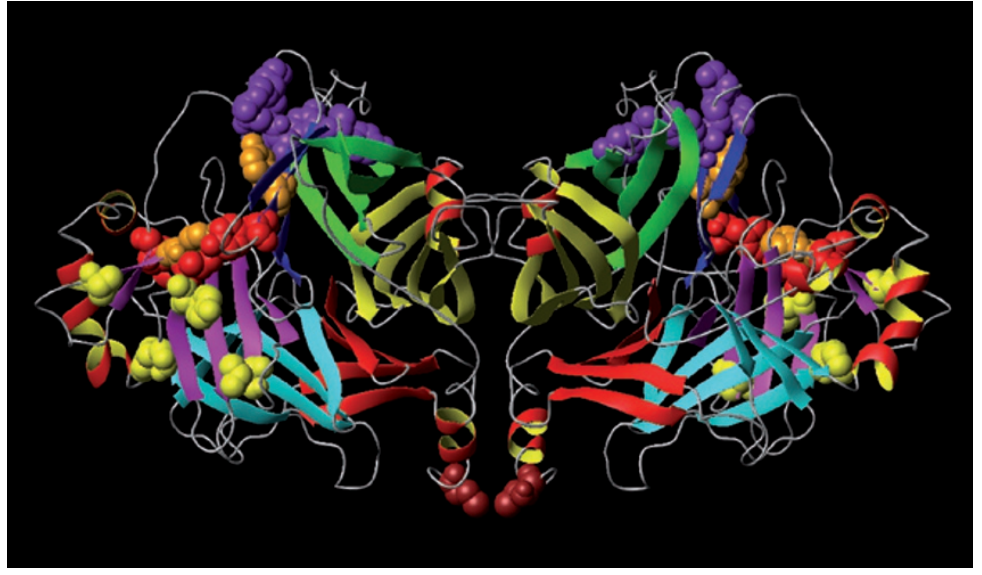

A ribbon representation of the crystal structure of the measles virus haemagglutinin protein homodimer; the residues that are important for signalling lymphocytic activation molecule (SLAM) recognition, EpR recognition and CD46 recognition are in purple, orange or red, and yellow, respectively. Image kindly provided by Vincent Leonard, Mayo Clinic, Rochester, USA

measles symptoms, but were unable to shed the virus into the airways, which supports the model that respiratory epithelial cells are not necessarily the initial targets of MV, but that infection of these cells is required for shedding and transmission.

The authors then sought to characterize this shedding-restrictive mechanism. Wild-type virus infected columnar epithelial cells that form tight junctions only when applied basolaterally and budded only from the apical side, a finding that is consistent with one-way epithelial crossing, in which epithelial cells enable shedding but are not the focus of initial infection. By contrast, the EpR-blind mutant virus did not enter epithelial cells basolaterally and did not infect epithelial cells. This confirms the hypothesis that EpR is a basolateral protein and that wild-type MV releases progeny through apical release from the epithelial barrier. This mechanism of shedding could explain why MV is transmitted through respiratory aerosols, and could also apply to other viruses that spread in a similar manner.

This enlightening study could force us to rethink our view of the MV infectious cycle. The authors also point out that this new understanding could have implications for the use of MV-based vectors in oncolytic therapies. More work is needed, however, to identify the basolateral EpR.

Gillian Young

ORIGINAL RESEARCH PAPER Leonard, V. H. . et al. Measles virus blind to its epithelial cell receptor remains virulent in rhesus monkeys but cannot cross the airway epithelium and is not shed. J. Clin. Invest. 20 June 2008 (doi: 10.1172/JCI35454) FURTHER READING Takeda, M. Measles virus breaks through epithelial cell barriers to achieve transmission.J. Clin. Invest. 20 June 2008 (doi: $10.1172 / \mathrm{JCl} 36251)$ 